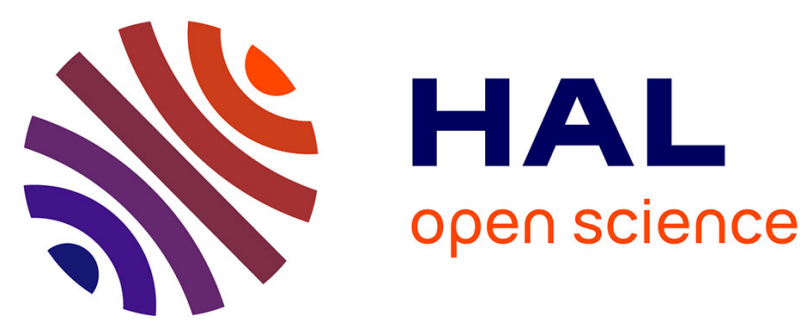

\title{
Sanctuaires et pratiques cultuelles : l'apport des recherches archéologiques récentes à la compréhension de la sphère religieuse des Gaulois
}

Patrice Arcelin, Jean-Louis Brunaux

\section{- To cite this version:}

Patrice Arcelin, Jean-Louis Brunaux. Sanctuaires et pratiques cultuelles: l'apport des recherches archéologiques récentes à la compréhension de la sphère religieuse des Gaulois. Gallia - Archéologie de la France antique, 2003, Cultes et sanctuaires en France à l'âge du Fer, 60, pp.243-247. 10.3406/galia.2003.3148. hal-01911707

\section{HAL Id: hal-01911707 \\ https://hal.science/hal-01911707}

Submitted on 9 Jan 2020

HAL is a multi-disciplinary open access archive for the deposit and dissemination of scientific research documents, whether they are published or not. The documents may come from teaching and research institutions in France or abroad, or from public or private research centers.
L'archive ouverte pluridisciplinaire HAL, est destinée au dépôt et à la diffusion de documents scientifiques de niveau recherche, publiés ou non, émanant des établissements d'enseignement et de recherche français ou étrangers, des laboratoires publics ou privés.

\section{(ㅇ)(1) $\$$}

Distributed under a Creative Commons Attribution - NonCommercial - NoDerivatives 44.0 


\title{
SANCTUAIRES ET PRATIQUES CULTUELLES
}

\author{
L'apport des recherches archéologiques récentes \\ à la compréhension de la sphère religieuse des Gaulois
}

\author{
Patrice ArCELIN et Jean-Louis BRUnAuX
}

Cette approche conclusive est bien plus modeste que nous ne l'avions souhaitée à l'amorce de ce travail. L'ampleur du dossier nous contraint, en quelques pages, à un simple résumé synthétique des acquis récents, avec la simple mention de quelqucs sites représentatifs ou d'allusions limitées à l'environnement sociopolitique des pratiques cultuelles concernées.

\section{DE NOUVEAUX ÉLÉMENTS POUR UNE LECTURE DIFFICILE}

Les chapitres de ce dossier sont riches d'enseignements sur l'avancée des recherches intervenues depuis une à deux décennies dans l'analyse des traces laissées par les différentes pratiques des cultes, de l'intimité de la maison aux vastes sanctuaires à vocation collective. À l'analyse des données factuelles récentes rassemblées par les intervenants, on perçoit les difficultés rencontrées par ce type d'exercice et les dangers de leur interprétation scientifique. De plus, toutes les régions ne sont pas également documentées et les synthèses révèlent d'importantes disparités dont les raisons sont variées. La plus évidente tient à la diversité des modalités d'investigation et à l'inégalité des prospections. Certaines régions ont vu se former des équipes dont l'un des sujets majeurs était l'étude des lieux de culte. Ailleurs, c'est le travail de chercheurs isolés qui a été fécond. Mais au total, de vastes zones demeurent des terrae incognitae. De ce fait, la programmation des recherches en France a encore beaucoup de chemin à faire, elle n'a même pas permis de commencer à combler les vides dans la carte nationale, ni d'harmoniser les modes d'investigation, à d'heureuses et récentes exceptions près.

En l'état actuel pourtant des pistes informatives se dessinent en Gaule, par-delà les spécificités macro- régionales. On en soulignera les dénominateurs communs même s'il convient de demeurer prudent sur la portée réelle des pratiques cultuelles entrevues. Les documents archéologiques, souvent limités dans leur ampleur ou la pertinence de leur valeur heuristique, sont fréquemment surinterprétés dans leur lecture, voire inconsciemment forcés dans leurs comparaisons interrégionales ou européennes. Inversement, la prise de conscience de cette attitude peut avoir pour conséquence de limiter la publication à un simple inventaire " à la Prévert », sans la vision culturelle d'ensemble s'articulant aux autres courants des évolutions sociales, économiques et politiques. Néanmoins, fouilles et études dans le domaine considéré permettent de reconnaître parmi les faciès régionaux et leur originalité certains caractères d'une cohérence cultuelle gauloise et, de ce fait, de leur réalité historique.

Comme on pouvait s'y attendre, toutes les régions de la Gaule ne fournissent pas les mêmes témoignages archéologiques des formes du culte, ni dans les mêmes proportions. Il en va ainsi des sanctuaires du type " aire fossoyée " qui sont abondamment représentés en Gaule, à l'exception (sans doute provisoire) des régions méridionales. Ces dernières, sans en être dépourvues, ont livré d'autres types d'aménagements cultuels comme les puits et des espaces bâtis à murs porteurs ou encore des délimitations en élévation d'aires consacrées. Sans renier les spécificités culturelles et les contraintes du milieu, on rappellera que cette répartition est également en Gaule la conséquence de la focalisation des investigations de terrain depuis plusieurs générations, recherche prédominante des enclos sur les territoires septentrionaux, des éléments bâtis et des objets en pierre dans les habitats groupés du Sud-Est. Par bonheur, bien des exceptions viennent déroger à ces orientations traditionnelles de la recherche de terrain. 


\section{DES CARACTÈRES CULTUELS COMMUNS AUX CELTES?}

On retiendra des analyses thématiques dans les interrégions françaises plusieurs dénominateurs qui paraissent relativement communs à l'ensemble de la Gaule celtique. Nous les énumérerons brièvement selon une double approche, la nature des lieux cultuels en rapport avec l'occupation des sols et celle des pratiques qu'ils recouvrent.

\section{DES LIEUX ET AMÉNAGEMENTS CULTUELS PRIVILÉGIÉS}

L'inventaire des lieux avec dépôts d'offrandes, reconnues en tant que telles, et l'analyse de leur organisation spatiale montrent que des caractères que l'on considéraient jusqu'alors circonscrits à quelques régions sont assez largement répandus, y compris sur la façade méditerranéenne du Sud-Est jusqu'alors marginalisée dans ce type d'approche.

- Presque partout, les lieux consacrés à la pratique des cultes sont disposés en majorité sur les territoires, hors des habitats groupés, même s'ils sont parfois peu éloignés de ces derniers.

- Il s'agit en premier lieu d'espaces naturels désignés par les divinités (hauteurs, rochers insolites, grottes, gouffres, rivières, étangs, forêts...) ou considérés comme majeurs dans la structuration territoriale des populations ou encore dans l'exploitation des ressources naturelles (limites de peuples, routes, mines, voies commerciales terrestres et maritimes...). La plupart de ces cultes trouvent leur rituel dans la continuité de ceux de la Préhistoire récente. Les aménagements des lieux caractérisés par des offrandes sont généralement très fugaces, limités à des fosses ou foyers, voire invisibles à la fouille.

- On considérera ensuite les aires aménagées et délimitées par des fossés et/ou palissades. C'est par excellence le mode généralisé de démarcation complète ou partielle d'une aire consacrée à une divinité (Les Sept-Perthuis à Saint-Malo, Bennecourt, Fesques, Mirebeau-sur-Bèze, etc.), aux défunts et aux pratiques honorifiques (ainsi l'enclos ovale de Ribemont-sur-Ancre ; ceux des Herbues à Vix et de Vestric-et-Candiac), aux réunions collectives avec banquets et libations (Le Brézet à Clermont-Ferrand, Les Genâts à Fontenay-le-Comte, Aix-en-Provence), mais également à toutes les activités profanes agropastorales (délimitations de parcellaires, enclos de fermes...). Petits enclos intérieurs, autels votifs en creux et couverts, fosses diverses ou puits chemisés peuvent participer des amėnage- ments complémentaires (Gournay-sur-Aronde, Le Brézet, Ribérolles à Rivières, etc.). D'autres techniques de délimitation sont également mises en œuvre, comme les haies vives ou les clairièrcs amćnagées (suggérées par les textes anciens).

- Enfin, apparaissent, dès le début du premier âge du Fer dans le Midi méditerranéen et pour l'essentiel dans la basse vallée du Rhône, des constructions disposées en des points sensibles des territoires et consacrées à des pratiques dévotionnelles singulières. Il s'agit de portiques à piliers et linteaux monolithes, version monumentalisée de réalisations en bois antérieures ou contemporaines (Mouriès, Entremont). Elles se maintiendront au second âge du Fer (Roquepertuse à Velaux). Ailleurs en Gaule, l'évolution de la complexité des enclos à autel en creux couvert conduira, par étapes, vers le type plus élaboré du fanum au cours du $\mathrm{I}^{\mathrm{er}}$ s. avant J.-C.

- Avec l'accroissement de son importance dans le mode organisationnel des territoires, son rôle économique, à terme politique et religieux, l'habitat groupé va engendrer ses propres aménagements cultuels ou récupérer à son profit ceux préexistants. Ces édifications paraissent dans un premier temps semblables à celles établies en milieu ouvert ou bien à faible spécificité architecturale. Ainsi les régions méditerranéennes de la Gaule, en fonction de leur développement précoce en ce domaine, montrent l'éclosion de pratiques cultuelles dans l'aire fortifiée du site dès la fin du premier âge du Fer. Elles se manifesteront ensuite, jusqu'au milieu du second, dans les pièces de l'habitation familiale par des fosses à offrandes ou d'objets étalés dans des espaces bâtis dédiés, mais peu différenciés des constructions de leur entourage. Ce n'est qu'à partir du III ${ }^{e}$ s., plus généralement aux $\mathrm{II}^{\mathrm{c}}$ et $\mathrm{I}^{\mathrm{er}} \mathrm{s}$. avant J.-C., qu'un développement plus monumental s'opère sous l'effet de stimuli culturels méditerranéens. L'adoption de la forme du temple gréco-italique en est la plus lisible. Elle participe, dans la basse vallée du Rhône, d'un bouleversement conceptuel $\mathrm{du}$ rôle politique et religieux des habitats majeurs (à l'exemple de Glanon). Mais d'autrcs formes plus celtiques émergent aussi dans les agglomérations ou à leur immédiate périphérie (comme l'aire cultuelle de Pech Maho, les puits et fossé de Rodez, l'enclos ovalisé de Constantine, le fanum de Nages, le nemeton en périphérie de Vaison-laRomaine...).

Dans le reste de la Gaule, c'est au cours des $\mathrm{II}^{\mathrm{e}}$ et $\mathrm{I}^{\mathrm{er}} \mathrm{s}$. avant J.-C. que l'on note la multiplication de fosses à offrandes (Goinet, Feurs, etc.) et la mise en place d'un habitat autour d'aires, de puits et de constructions cultuelles, qui concrétisent de ce fait la liaison du politique et de l'économique au religieux (Acy-Romance, Bibracte, Aulnat, Le Brézet, Clorent, Vienne, Toulouse...). 


\section{DES PRATIQUES DÉVOTIONNELLES ET PROPITIATOIRES RÉPANDUES}

C'est surtout l'offrande qui de par sa conception même s'affirme comme le rite principal des Celtes mais dont les formes et les objets qu'il concerne demeurent encore imprécis. Par une interprétation trop hâtive, par manque d'attention, bien souvent sont confondus l'offrande ellemême avec les restes sacrificiels ou avec le matériel utilisé pour le sacrifice et le banquet qui l'a suivi.

- Les dépôts d'objets, et en particulier ceux n'ayant pas de rôles directement fonctionnels dans la préparation de banquets, constituent par excellence les éléments de l'offrande aux divinités, qu'elles soient majeures ou topiques. Ils sont présents dans l'ensemble des lieux cultuels, tant sur les territoires que dans l'habitat à l'exception, semble-t-il, des portiques méridionaux précédents. Les objets consacrés, qu'ils soient modestes (fragments métalliques remployés ou céramiques retaillées) ou précieux (ainsi l'or et l'argent des Tectosages à Toulouse) sont déposés à même le sol, exposés, enfouis ou immergés. Les composantes métalliques (pièces d'armement, de vaisselle ou de l'outillage ; bijoux ; monnayage surtout à l'orée de la romanisation...) sont les traceurs indiscutables de ces consécrations, qu'il y ait eu ensuite destruction rituelle ou non. Ces objets exposés peuvent être classés dans la catégorie que les Grecs appellent anathémata. On a souligné le caractère sacré de ces lieux et leur pouvoir de protection des richesses.

D'autres types d'objets peuvent avoir été offerts en tant que tels (ainsi les céramiques peintes de La Bauve à Meaux, des bois de cervidé ou certaines des stèles en pierre d'Armorique ou du Midi), mais généralement vaisselle céramique, amphores vinaires, dolia, bétyles ou meules de mouture ne sauraient être confondus avec l'offrande ellemême. Ce sont les vestiges des rituels, l'accompagnement utilisé pour le sacrifice et son souvenir, ainsi que pour le banquet et les libations qui l'ont suivi.

Les travaux de ces dernières années dans l'ensemble de la Gaule ont bien montré la nécessité de séparer, selon leurs composantes, les reliquats découverts dans les fossés des enclos quadrangulaires de la fin du second âge du Fer. Ainsi ont été mis en lumière des pratiques essentiellement de nature festive et politique, mais certainement associées à des libations. Ces « enclos à banquets » et « fossés à amphores ", caractérisés essentiellement par des accumulations de conteneurs vinaires, des traces de cuveaux ou de dolia et des restes de préparation alimentaire, trouvent une forte résonance dans les témoignages de la commensalité antique. Ils sont largement répandus dans l'ensemble de la Gaule, tant hors des agglomérations (Arnac-la-Poste, Trouy près de Bourges, Le Brézet, Aix-en-Provence) qu'en relation plus directe avec elles (ainsi sur l'oppidum de Corrent, Lyon). Une plaque en calcaire gravée de Cavaillon montre une scène de ces libations vers la fin du premier âge du Fer. Des actions de cette nature ou proches sont également pressenties dans les rites domestiques (Aulnat, Martigues).

- Les sacrifices d'animaux, presque exclusivement domestiques, sont généralisés en Gaule, dans les grands sanctuaires (Gournay-sur-Aronde, Fresques, Bourges, Muron, Corent...), mais aussi les lieux cultuels plus modestes en habitat (Verdun-sur-le Doubs, Graveson, Gaujac), voire dans les pratiques domestiques (Martigues). L'abattage isolé ou en nombre de chevaux, près d'inhumations collectives ou en relation directe avec ces dernières, dénote d'autres formes sacrificielles hors de sanctuaires structurés (Vertault, Gondole au Cendre, Le Brézet). Dans ces derniers, l'autel en creux tient une grande place dans les pratiques collectives. Il comprend deux formes qui peuvent être réunies. La cavité qui est comparée à celle du culte chthonien du monde gréco-romain, et le foyer qui permet la cuisson des viandes et de la fressure de l'animal sacrifié. L'iconographie révèle quelques-unes des attitudes des dévots portant l'offrande d'un petit animal (Entremont).

- La découverte de restes humains dans plusieurs sanctuaires du Nord et du Centre-Ouest de la Gaule, mais également, ici comme ailleurs, dans des silos abandonnés ou en habitat, a généralisé l'idée du sacrifice humain, le seul à avoir été décrit succinctement par les auteurs antiques. On ajoutera à cette liste l'existence de restes incinérés disposés exceptionnellement en habitat. Il convient en réalité de séparer ici deux modes bien différents : l'immolation d'individus dans le cadre des rituels stricts d'un sanctuaire, quelles qu'en soient les modalités, et la manipulation de corps ou de parties de ceux-ci, disposés dans les fondations d'une fortification, d'une habitation ou en silos rebouchés. Laissant de côté ici les rites de victoire (sous la forme de têtes coupées exposées ou de corps composant un trophée, comme à Ribemont-sur-Ancre ou à Mœuvres), il s'agit clairement dans le premier cas de l'offrande suprême et exceptionnelle à une divinité. Pour le reste, et hors cas de relégation, l'interprétation sera de nature propitiatoire, même si les modalités demeurent délicates à cerner : offrandes possibles pour la bonne conservation des récoltes, protection de l'habitat et de leurs occupants par les reliques de personnages majeurs. Les corps en fosse d'Acy-Romance, ceux de Mozé-sur-Louet ou encore ceux des avens de Plérimond à Aups et Bernard à Vallauris, restent problématiques et révèlent l'étendue des lacunes de nos connaissances en ce domaine. Même bien attesté, le rite du sacrifice humain est toujours globalement mal documenté par l'archéologie. 
On rattachera à ces rites de manipulation des corps humains le traitement de crânes honorés de leur seul masque facial parfois, certainement reconstitué au naturel et peint (entrées de sanctuaires, comme envisagés à Montmartin ou Gournay-sur-Aronde ; présentation dans les encoches des portiques cultuels peints à Glanon, Roquepertuse ou Nîmes par exemple). De même, l'enclos arrondi de Ribemont-sur-Ancre, par son autel, son foyer et ses stèles du souvenir, peut être assimilé à une forme d'hérôon, avec la pratique du banquet funéraire. Mais ici les corps ne sont pas encore réduits à l'état de reliques, plutôt exposés au décharnement des oiseaux de proie (selon des rites relatés dans l'Antiquité).

Au-delà des ces témoignages singuliers, une autre pratique est désormais mieux documentée en Gaule tant par les recherches de terrain que par l'épigraphie, celle de l'héroïsation de personnages majeurs, fréquemment des guerriers. Le réexamen de la statuaire gauloise, essentiellement anthropomorphe, en bois ou en pierre, ne la rattache pas à des représentations de divinités, mais à celles d'ancêtres ou de contemporains héroïsés, dans le cadre de cultes familiaux ou collectifs. Cette iconographie, dont le degré de réalisme varie selon les régions et leurs antécédents culturels (plus marqué vers la côte méditerranéenne qu'au nord), débute dès la transition bronze-fer ou peu après, tel le " groupe des bustes» du Languedoc oriental. Comme aux Herbues de Vix, vers l'extrême fin du premier âge du Fer, il est possible qu'une large part de ces sculptures ait été liée à des enclos similaires, disposés à proximité d'une sépulture isolée ou d'une nécropole familiale. Au second âge du Fer, la classe aristocratique paraît avoir pleinement récupéré cette pratique pour conforter son rôle et structurer son clientélisme. Les statues de Saint-Symphorien à Paule, comme celles rassemblées de façon circonstancielle à Entremont ou encore celles plus anciennes peut-être toujours exposées à Roquepertuse au $\mathrm{III}^{\mathrm{e}} \mathrm{s}$., suggèrent une présentation pour l'essentiel établie au sein de sanctuaires domaniaux. De leur côté, les habitats groupés feront davantage appel aux restes inhumćs ou incinćrćs dc héros protecteurs (Mailhac, SaintPriest et Gorges de Loup à Lyon, Pech Maho à Sigean), ou tardivement à une récupération iconographique valorisante (Glanon et La Cloche aux Pennes-Mirabeau). À la fin du second âge du Fer, des sculptures peuvent être également disposées dans des dépôts (Levroux, Châteaumeillant).

\section{DES SPÉCIFICITÉS CULTUELLES INTERRÉGIONALES}

Nous avons souligné précédemment un certain nombre de caractères qui apparaissent propres à de vastes régions de la Gaule. Nous en retiendrons seulement trois bien établis :

- au second âge du Fer, les vastes sanctuaires collectifs sont plus spécifiques au Nord el à l'Ouest de la Gaule (Gournaysur-Aronde, Fesques, Estrées-Saint-Denis, Saint-Maur...), avec une pratique des sacrifices humains, qui se raréfie également plus au sud où les dépôts en puits apparaissent plus usuels. Quant à la façade méditerranéenne, une partie de ses lieux cultuels ne commenceront à se monumentaliser que sous l'influence des apports gréco-italiques, après le milieu du II ${ }^{\mathrm{e}}$ s. avant J.-C. (Glanon, Nîmes) ;

- conjointement, la nature des offrandes se différencie entre ces mêmes régions. L'armement est fréquent dans les régions septentrionales et du Centre-Ouest (aux sites précédents, on ajoutera ceux de La Bauve, Saint-Jean-Trolimon, Saumeray, Puy de Dôme, Muron, Corent...). Dans le Sud-Est ou le Centre, concernés tôt par le commerce méditerranéen, les importations de céramiques et pour l'essentiel les conteneurs vinaires dominent largement (puits du toulousain ou de la région de Rodez, Aix-en-Provence, Lyon, Mirebeau-sur-Bèze, Châteaumeillant...) ;

- la limitation des fouilles d'ampleur dans l'habitat groupé gaulois ne permet pas de juger correctement de l'intégration comme de l'importance des lieux cultuels avant La Tène finale. Le cas d'Acy-Romance est pour l'heure bien isolé. La façade méditerranéenne, par la précocité du développement de ses habitats groupés et fortifiés an premier âge du Fer, permet de saisir les pratiques liées à la protection de l'enceinte et de ses accès. Les petites aires de culte accolées à la fortification sont assez récurrentes jusqu'à la romanisation (Graveson, Gaujac, La Cloche...) et se maintiendront parfois même après l'abandon du site (Ambrussum, Nages).

\section{DU RELIGIEUX DANS LA SOCIÉTÉ CELTIQUE}

L'étude archéologique des formes du culte a incontestablement ouvert de nouvelles voies à la recherche sur les structures de la société. Des analyses sur l'évolution de la morphologie des lieux cultuels et des pratiques qui s'y rattachent transparaît l'image, plus ou moins précise selon les régions, de transformations perceptibles par ailleurs dans la vie des populations de l'âge du Fer. Au-delà d'une forte pérennité des croyances essentielles et des démarches cultuelles individuelles ou familiales récurrentes, ce sont les actions collectives qui vont le plus évoluer dès l'extrême fin du premier âge du Fer, plus généralement dès le III ${ }^{e} \mathrm{~s}$. Elles reflètent les étapes de la structuration des peuples sur leurs territoires (frontières, organisation politique, place des 
habitats groupés puis des protovilles), la montée en puissance de la classe aristocratique (et de son besoin constant d'affirmation) comme les évolutions socioculturelles qui se font jour au sein des acteurs politiques et économiques confrontés aux sollicitations croissantes des apports méditerranéens à partir du II ${ }^{\mathrm{c}} \mathrm{s}$. avant J.-C. (pour l'essentiel, le Midi, le Centre et le Centre-Est).

Dans la première partie de l'âge du Fer, les lieux cultuels demeurent relativement modestes, limités à une fréquentation familiale (comme les grottes ou les enclos parafunéraires) ou plus communautaires (les portiques à reliques du Midi). Le culte « princier " du premier âge du Fer, à forte connotation funéraire, n'exigeait pas un personnel particulier ou nécessitant une formation spécifique. Le petit enclos quasi familial des Herbues, découvert auprès de la tombe princière de $\mathrm{Vix}$, suggère plutôt que les rites pouvaient être exécutés dans la sphère intime du prince, peut-être par lui-même. C'est au III ${ }^{\mathrm{e}} \mathrm{s}$. avant J.-C. (plus tardivement sur la façade méditerranéenne) que se produisent de grandes mutations, avec la multiplication de lieux de culte d'importance et plus monumentaux. Ils reproduisent des plans similaires, obéissant à des règles astronomiques avec la réalisation de rites complexes et se déroulant sur des périodes très longues. Il en découle la nécessité d'un entretien des structures, la mise au point de calendriers et la conservation d'archives sacrées qui supposent la mise en place progressive d'un clergé puissant et hiérarchisé. Ce dernier se décomposait vraisemblablement en spécialités, prêtres, devins, sacrificateurs. Il ne paraît pas aventureux de voir, au moins dans une partie de ces hommes, des druides, au sens où l'entend César. La qualité de druide (l'équivalent de «l'augure » latin) n'est pas assimilable à une profession mais correspond plutôt à un titre, un privilège. Ils s'opposaient à la popularisation des rites et de la connaissance des dieux. Ce que César nous dit de leur savoir jalousement gardé s'accorde bien avec l'image qui nous est donnée par les sanctuaires hermétiquement fermés où régnait l'aniconisme le plus pur.

Cantonné initialement aux territoires, les sanctuaires vont s'associer aux bourgades par étapes. Très modestes à la fin du premier âge du Fer près des côtes méditerranéennes, ils ne prendront une réelle ampleur qu'au $\mathrm{II}^{\mathrm{e}} \mathrm{s}$. (AcyRomance), et plus généralement à partir du $\mathrm{I}^{\mathrm{er}} \mathrm{s}$. avant J.-C. dans les plus vastes agglomérations et protovilles en formation (ainsi à Glanon dans le Midi, Bibracte, Besançon et Mandeure en Gaule interne).

Parallèlement, le développement probable de sanctuaires privés sur des propriétés rurales, avec ou sans iconographie héroïque conservée (à l'exemple de Montmartin ou de Paule dans le Nord et l'Ouest, des éléments de la statuaire rassemblés à Entremont dans le Midi), souligne la prégnance politique de ces domaines familiaux aristocratiques et le rôle joué par le facteur de l'héroïsation dans la pérennité de leur statut. De même la multiplication des " enclos à banquets " révèle l'implication ostentatoire de cette classe dans les manifestations collectives à libation et l'impérieuse nécessité pour elle de conforter son clientélisme par le jeu des somptueux banquets relatés par les textes anciens.

Ces derniers enclos, lieux d'assemblées, de banquets ou de tribunaux, sont autant d'aménagements collectifs qui témoignent d'une civilisation développée, aux institutions en retard d'un ou deux siècles sur celles des cultures gréco-latines. Ainsi se vérifie dans les domaines de la politique et des pratiques sociales les plus diverses ce qu'on pouvait présumer de la découverte, il y a vingt-cinq ans, des premiers sanctuaires celtiques et des sacrifices qui s'y déroulaient. 